IWSS

Information Systems

INS $\begin{aligned} & \text { INformation Systems } \\ & \text { Cuypers Meets Users: Implementing a User Model } \\ & \text { Architecture for Multimedia Presentation Generation }\end{aligned}$

Suzanne Little, Lynda Hardman

Report INS-E0306 November 14, 2003 
CWI is the National Research Institute for Mathematics and Computer Science. It is sponsored by the Netherlands Organization for Scientific Research (NWO).

$\mathrm{CWI}$ is a founding member of ERCIM, the European Research Consortium for Informatics and Mathematics.

CWI's research has a theme-oriented structure and is grouped into four clusters. Listed below are the names of the clusters and in parentheses their acronyms.

Probability, Networks and Algorithms (PNA)

Software Engineering (SEN)

Modelling, Analysis and Simulation (MAS)

Information Systems (INS)

Copyright (C) 2003, Stichting Centrum voor Wiskunde en Informatica

P.O. Box 94079, 1090 GB Amsterdam (NL)

Kruislaan 413, 1098 SJ Amsterdam (NL)

Telephone +31205929333

Telefax +31205924199 


\title{
Cuypers Meets Users: Implementing a User Model Architecture for Multimedia Presentation Generation
}

\begin{abstract}
With the rapid growth of interest in the Internet as a means for accessing multimedia presentations for education, entertainment and commerce, comes a corresponding need for systems to supply automatically generated, personalised presentations. Multimedia is a rich and complex genre of resources and the interrelated effects of content, style and structure ensure that automatic presentation generation is in itself a complicated and challenging task. Integrating a model for user personalisation adds a further layer of complication and ensuring that the requirements of user, supplier and platform are all met is a demanding undertaking. This project investigates the influence of information about a user in the process of generating a multimedia presentation. As a result an architecture taking into account these trade-offs is proposed. To evaluate this combined architecture, a framework has been implemented which adjusts colour choices based on the different influences involved. This paper describes the integration of a user modelling approach within an existing system architecture, discusses some of the issues involved in applying user modelling to multimedia presentation generation and describes the prototype implementation and how it addresses some of these issues.
\end{abstract}




\title{
Cuypers Meets Users: Implementing a User Model Architecture for Multimedia Presentation Generation.
}

\author{
Suzanne Little \\ ITEE Dept, University of Queensland and \\ Distributed Systems Technology Centre (DSTC) \\ slittle@dstc.edu.au \\ Lynda Hardman \\ Centrum voor Wiskunde en Informatica (CWI), Amsterdam \\ Lynda.Hardman@cwi.nl
}

\begin{abstract}
With the rapid growth of interest in the Internet as a means for accessing multimedia presentations for education, entertainment and commerce, comes a corresponding need for systems to supply automatically generated, personalised presentations. Multimedia is a rich and complex genre of resources and the interrelated effects of content, style and structure ensure that automatic presentation generation is in itself a complicated and challenging task. Integrating a model for user personalisation adds a further layer of complication and ensuring that the requirements of user, supplier and platform are all met is a demanding undertaking. This project investigates the influence of information about a user in the process of generating a multimedia presentation. As a result an architecture taking into account these trade-offs is proposed. To evaluate this combined architecture, a framework has been implemented which adjusts colour choices based on the different influences involved. This paper describes the integration of a user modelling approach within an existing system architecture, discusses some of the issues involved in applying user modelling to multimedia presentation generation and describes the prototype implementation and how it addresses some of these issues.
\end{abstract}

\section{Introduction}

Personalising the interface between man and machine has long been an area of interest and research. With the increasing popularity of the World Wide Web exciting new areas for applying personalisation techniques have emerged and with them new issues to be addressed. The rich re- sources now available include many non-textual formats and as methods for retrieving and presenting this information are developed, a desire for increasing user support is born. A solution is, by the very natures of both multimedia and users, complex and often incomplete. This project investigates a possible solution under development by $\mathrm{Su}-$ sanne Loeber ${ }^{1}$ and applies it to a prototype system for automatically generating multimedia presentations.

The field of user modelling seeks to improve the manner in which users and computers interact and communicate by understanding and recording user characteristics. A model is developed which allows the system to make predictions about the user, their requirements and behaviour. Once built, this model can assist the system in adapting its interface to the user's goal, skill level, domain knowledge and system requirements among other things. The result of this personalisation is an increase in user satisfaction and success in using the system.

Historically user models have been applied in one of two ways. Either the model has been crafted specifically for a system and is not easily transferable to another system (for example the context sensitive user support provided in the Microsoft Office suite [15]) or the model has been built into an independent system which can provide support to various types of applications (for example the BGP-MS system [10]). AVANTI [5] is an example of a hypermedia system implementing user modelling focusing on users with special needs (eg. low bandwidth, physical restrictions). The models used in these systems may therefore be very different in content, purpose and longevity.

The use of user modelling to provide personalised computer interaction is not without difficulties. These include

\footnotetext{
${ }^{1}$ Susanne Loeber (Susanne.Loeber@tue.nl) is currently a PhD student with the University of Eindhoven.
} 
extracting information from the user, inferring and applying generalisations about the specific user from available information and supporting sufficient adaptability and flexibility, both within the model and the application, to make adequate use of the information [11] [16] [4]. Kobsa discusses the "paradox of the active user" [8] and notes that while personalisation may be something that users appreciate, they are unlikely to take time to provide information that they see as unnecessary for their final goal. This difficulty in extracting information is just one example of some of the problems faced when trying to characterise a user and develop an accurate and viable model.

The rise of the Internet has opened up a new field for user modelling - adaptive hypertext and hypermedia. Whether adapting to a user's knowledge level (KN-AHS [9] [2]) or simulating individual 'attention' from an e-commerce site [8], the application of user modelling to the World Wide Web and its constituent technologies is ripe with possibilities. Unfortunately it also comes with many of the same problems that user modelling has in the more traditional areas of application.

Retrieval of accurate and timely information about the user is a recognised problem in user modelling. This difficulty is exacerbated in a web-based environment since interaction with the user is often transient. Loeber's approach, described in [12] [13] [14], seeks to overcome some of these limitations through a user-centered, context-driven approach to modelling and by focusing on the relationship between the user and the author of the presentation or site, to capture and manipulate the user's motivation, ability and opportunity levels. By utilising concepts from Persona Theory [3] and the Situated Action Framework [17], the interaction with the 'visitor' to a site begins with a general user profile before interaction allows refinement of the profile to enhance the user's motivation, ability and opportunity levels.

The focus on the user's levels of motivation, ability and opportunity (MAO), as applied by Loeber, is based on a model from Hoyer and McInnis [6].

Motivation is the user's desire to complete their plan or attain their goal. The ideal state is to maintain a high level of personal relevance and consistency with values/cultural beliefs and keep perceived user risks and inconsistency with user attitudes low.

Ability is the extent to which the user has available resources with which to complete their plan. Keeping a satisfactory ability level includes factors such as knowledge, skill level, money and physical abilities.

Opportunity rates the elements which could hinder the user from completing their plan such as time available and distractions (both within the presentation or site and externally). Optimal interaction occurs when there is limited distractions and therefore a high opportunity.

The user and author can be viewed as two personas interacting in roles to fulfil certain needs which can be either social or non-social and of functional, symbolic or hedonic natures. By modelling their interactions and considering the context within which it takes place, a mechanism is available to find compromise between conflicting design requirements. The impact of design decisions upon the user's motivation, ability and opportunity levels and the rules which describe this impact, form the key to Loeber's approach. They seek to provide a means to measure, and therefore discuss, the affect of implementing a particular decision on a particular user.

Some important terms which must be explained at this point are the concepts of user, visitor and author. The visitor in Loeber's model refers to specific information about the person currently interacting with the system. A user is anyone who visits the web site. Users are captured in a collection of user profiles, stereotypes containing relationships between certain combinations of user characteristics and rules describing the MAO impact of implementing particular design decisions. The author is the person or entity (often a company) who created and maintains the site. Therefore the author is concerned with issues such as corporate image, successful sales or achieving educational goals, depending upon their purpose for the site.

The general process of Loeber's approach is as follows. The visitor is initially assigned a generic user profile built around the author's preferences and beliefs. As the visitor interacts with and navigates through the site, inferences about their possible goals are made and a user profile is assigned to them. This is based on the navigation path which they follow through the site. The assumption is that people who follow the same path have similar characteristics and goals and therefore are assigned the same user profile. As the visitor continues to interact with the site more specific user profiles are assigned to reflect the navigation choices they have made. With each new profile assigned to the visitor, the appearance, style and structure of the pages may be updated to reflect the new profile. Information on the visitor's platform and previous interactions, where available, are also incorporated into decisions.

Fig. 1 shows this approach, combined with the Standard Reference Model for Intelligent Multimedia Presentation Systems (SRM IMMPS) [1], to illustrate application in the area of dynamic creation of web-based multimedia presentations.

The four key extensions to the SRM IMMPS that Loeber's approach supplies are the Context Model Expert, User Model Expert, Discourse Expert and the Optimisation Layer. 


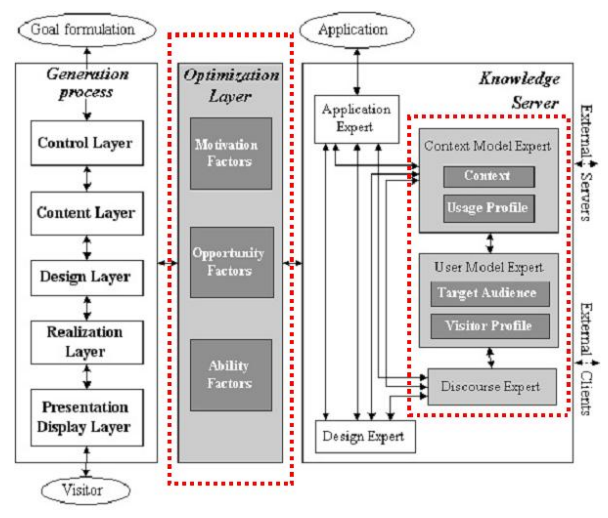

Figure 1. Loeber's Model [13]

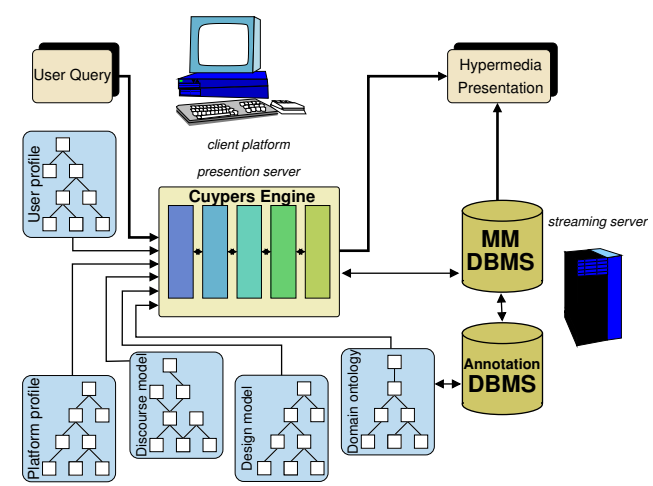

Figure 2. Information sources used by the Cuypers adaptive hypermedia engine [20]
The Context Model Expert, an extension of the SRM IMMPS' Context Expert, contains two parts. First the Context which generates knowledge about content, how the presentation components have been gathered and combined and tracks the navigation path and interaction with the user. The second part, the Usage Profile, contains knowledge about the user's device profile - platform and other specifications related to displaying the presentation.

The User Model Expert, an extension of the SRM IMMPS' User Expert, also has two sections. The first, Target Audience Model, encompasses the general user model and the collection of user profiles containing the characteristics of the different users. The second part, the Visitor Profile, holds knowledge about the visitor.

The Discourse Expert is added to the knowledge server from SRM IMMPS to contain knowledge about the discourse style elements and interaction rules. Information on how the two personas, author and visitor, interact within the structure of the presentation is held here.

Finally the Optimisation Layer is used to solve conflict between the information coming from the different knowledge experts by attempting to find the optimal balance of motivation, ability and opportunity levels.

By this combined approach of modelling interaction between an author and a user and using the interaction to refine and select user profiles, Loeber seeks to overcome the problem of incomplete information about a user and to enhance the effectiveness of the presentation for all of the participants. This project aims to encapsulate in a framework Loeber's user modelling approach and the Cuypers presentation generation engine, a prototype for automatically generating multimedia presentations.

Cuypers [18] is a research prototype system developed at CWI to experiment with the generation of web-based hypermedia or multimedia presentations. It draws content from semi-structured multimedia databases and, given a rhetorical structure and set of design rules, the system generates a presentation that adheres to the limitations of the target platform. Simple examples of user adaptation can be found already in the Cuypers system. A profile describing the system capabilities is currently used to adapt presentations to varying screen size and bandwidth. Some general adaptation of content depending on the user's chosen knowledge level is also possible.

Many of the difficulties in the automatic generation of multimedia presentations are related to the interdependencies between content, style and structure [19]. For example, content can influence both the overall style of a presentation and the structure chosen to present it. A collection of images may have a common colour which matches well with a certain background. The sizes of the images may alter the layout chosen in an effort to present the information most effectively and the presence or absence of pieces of information in the content can change the structure of the presentation. Therefore design decisions can have far-reaching and complex consequences which need to be taken into account in a system that generates presentations automatically.

Since the Cuypers architecture, shown in Fig. 2, is derived from the SRM IMMPS model then extending it to encompass Loeber's user model architecture is not an illogical step to take. Equivalence between the participants in the Cuypers Knowledge Server and the Knowledge Server for Loeber's model shown in Fig. 1 is roughly as follows. Domain Ontology is part of Application Expert, Design Model is part of Design Expert, Discourse Model is part of the Discourse Expert, Platform Profile contained in the Context Model Expert and User Profile is contained in the User Model Expert.

The remainder of this article looks at the issues involved in incorporating user modelling into the Cuypers architecture. It discusses some of the problems with applying Loeber's model to an implementation and describes the imple- 


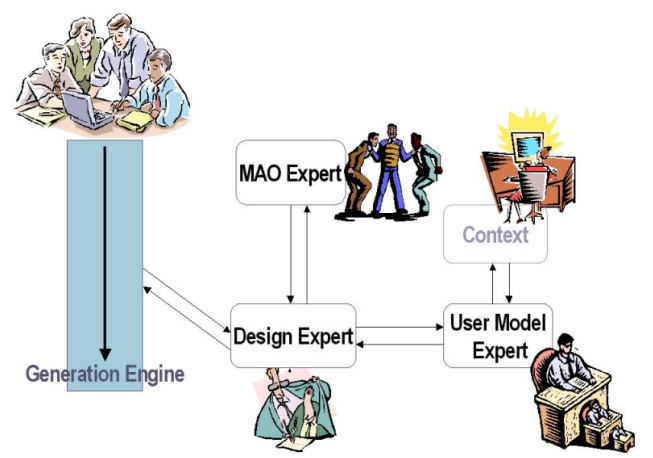

Figure 3. Architecture

mentation that has been done so far. Finally it examines the direction for future work.

\section{Architecture}

This section describes the implementation of the model and also presents the way in which some of the problems discussed in the previous section were addressed. The aim of this implementation was to provide the groundwork for the influence of user characteristics over the style, content and structure of automatically generated presentations. Due to the limited timeframe available for this project some boundaries were placed. It was assumed that information would be available from the user in some manner yet to be implemented and that rules and guidelines for design decisions based on user characteristics could be developed. The aspect of colour was selected for initial implementation as it provided an option which had obvious effects and some rules of application predefined.

The goal of the implementation was to provide a module that, when requested, supplied a foreground and background colour to the Cuypers generation engine based upon user characteristics. To apply Loeber's model to achieve this goal, the User Model Expert, Design Expert and some decision making ability based upon the MAO principles needed to be written. Three modules were created, the User Expert, Design Expert and MAO Expert and are described in detail below.

Fig. 3 shows the flow of information between the modules and how they interact with the existing system. A conversation between the current components of the user modelling implementation could sound something like this.

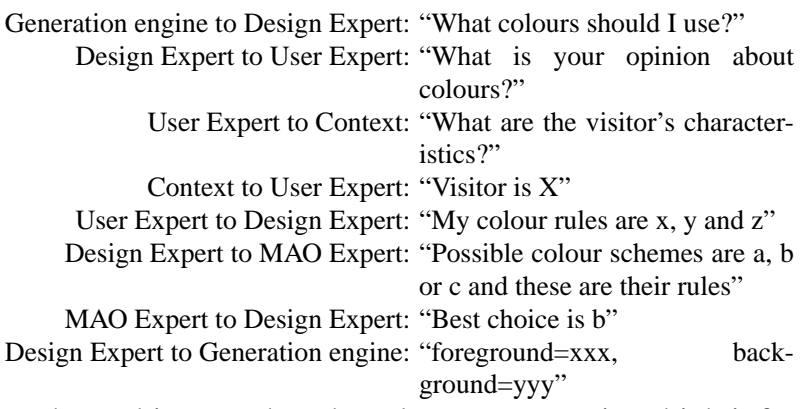

The architecture has three key structures in which information is stored: user profiles, rules (currently design rules for colour) and abstract values (currently describing colour values and schemes).

User profiles are lists containing pairs of characteristic:value which describe the characteristics of visitors who fit this profile. Each profile needs an id (for internal use only) and a value for each characteristic. These profiles then need to be associated with a list of rules for each design decision, ie. colour, text size, presentation speed etc.

Rules contain the persona or perspective (either user or author), an attribute (colour) value (an absolute HueSaturation-Luminescence, HSL value or abstract value) and values for Motivation, Ability and Opportunity. The MAO values can be either positive, negative or neutral depending on how predicted use of the attribute value will effect the visitor.

For the currently implemented design feature, colour, the abstract values come in two forms. First is a value which allows colours to be relative rather than absolute. Rules can then refer to concepts such as red or bright rather than precise colour definitions and rely on the design expert to understand the meaning when it is required. The second form is a scheme which describes a colour combination - a complete colour solution of foreground and background colour. Rules can then be written about the combined effect of two colours.

The goal of the User Expert is to store the user profiles and rules for colour preferences for each profile and to take the incoming information known about the visitor and use it to select the most appropriate user profile. This selection utilises some of the principles of MAO to ensure that the profile selected is the most supportive of the user. Selection is performed by means of a ranking system across a comparison of the user and visitor characteristics. Each characteristic of the visitor is compared with a stored user profile and its value for that characteristic and given a difference rank dependent upon how closely the values match. The rank is decided based upon the predicted effect upon the visitor's motivation, ability and opportunity.

For example, a visitor profile with a knowledge level of beginner and a user profile with the same value would be assigned a difference rank of 0 . If the user profile had a knowledge level value of expert then the difference rank 
would be higher (currently 5) as it would be more debilitating for a beginner to view a presentation designed for an expert than one designed for a beginner. On the other hand, if the visitor was an expert and the user profile was for a beginner then the difference rank would be 3 since this difference does not have as negative an impact.

The idea behind the difference ranking is that, when selecting the most appropriate user profile, the profile chosen should hopefully increase the user's motivation, ability and opportunity levels but, at worst, should not have a negative impact but attempt to maintain stable levels.

The Design Expert contains the definition of colours, the schemes detailing various possible combinations and any general design rules from the author (eg. green background is bad, corporate colours are $\mathrm{x}$ and $\mathrm{y}$ ) written in the same format as rules provided from the user expert. The purpose of the design expert is to encompass all specific information about the design attributes (in this case, colour) which includes abstract representations of colours and colour classes (eg. red, bright, dark), methods for translating these classes to the absolute values required by the generation engine and methods for choosing which rules apply to a particular value (eg. rules for bright colours also apply to red since red is classified as a bright colour).

By encapsulating the values in an abstract structure, the design expert infra-structure can be extended to handle other, more complex, design attributes. For example, the size and style of text may have an impact on the colours selected to display it. By storing this information in a scheme as a combination of factors, rules can be written that apply to this scheme. As long as the design expert is able to find all rules which apply to a particular solution and to turn the abstract representation of the solution into a format suitable for the generation engine then any design attribute can be recorded in this abstract form and decisions about its effectiveness based on recorded rules can be made.

The design expert also has another important function. The final result requires a complete solution, a colour scheme, which currently consists of a foreground colour and a background colour. To create these schemes a method for discovering complementary colours is required. Work being conducted in parallel with the user modelling project ${ }^{2}$ has been investigating ways of coding rules about colour combinations into a system which can then provide appropriate matching colours. A prototype exists which returns a background colour when supplied with a foreground colour in HSL format. The colour matcher is used to create schemes from colours which have positive rules associated with them. These schemes are then provided to the MAO expert as possible solutions together with any applicable rules about the colours involved.

\footnotetext{
${ }^{2}$ Work on developing and implementing colour design rules was conducted by Amit Manniesing (Amit.Manniesing@cwi.nl) at CWI
}

The MAO Expert acts like a mediator to balance the rules provided by the user and design experts and select the scheme (solution) which gives the best results for the visitor's motivation, ability and opportunity. It takes a list of rules about a particular scheme known only by its identifier. First the rules are combined to produce a value for each of motivation, ability and opportunity which indicates the predicted impact as either positive, negative, neutral or unknown. If there is disagreement between any rules, that is one gives a positive value and the other a negative value, then the result is listed as unknown as it is not possible to say what the impact could be. Once this combination has been performed two schemes are compared with each other and, based on the MAO values, one scheme is indicated as being a better solution than the other. When doing this comparison the general rule applied was that the most important aspect was a user was able to use the system (Ability), then that they wanted to use the system (Motivation) and finally that they had the optimal opportunity to use the system (Opportunity).

This implementation has describes a method for implementing MAO and describing the impact of design decisions. A module to compare and rank solutions based on the MAO concepts has been written. By creating compound, abstract values to store the design solutions (in this case the colour schemes which allowed the combined impact of two colours to be described) a partial solution has been found to the complexity issue. An approach to matching user profiles based on MAO principles has also been developed and implemented using a difference rating to find the solution which will cause the least negative impact. Overall a base for further expansion has been laid. The next section discusses some of the issues which were considered when applying this approach.

\section{Discussion}

Incorporating user modelling into multimedia presentation generation raises a number of issues. Some of these, such as extracting information from the user, are common to all attempts at user modelling and adaptation while others are caused by the unique qualities of multimedia.

\subsection{User Profiles}

To overcome the issue of lack of available information about a user, Loeber's approach assumes the existence of an extensive collection of user profiles, stereotypes, in an attempt to provide sufficient coverage of all user types. The use of the term stereotype often has negative connotations. It generally brings to mind a sense of rigidity and generalising, a short-sighted view unable to adapt to the reality of a situation. While perhaps there is some truth to this 
view in human terms, when applied to creating stereotypical user profiles for use in user modelling, stereotypes are a useful and credible means to deal with the lack of information problem. In this instance, the number of user profiles available increases the chance that an adequate match can be made and the adaptability inherent in Loeber's approach improves the matching odds still further.

Unfortunately to fully realise coverage of all potential user types, the collection of user profiles coded into Loeber's model must be complete and organised. This entails a large amount of work from the human designers in creating and implementing the profiles. Not only must decisions be made as to the content of the profiles, that is the characteristics recorded, but the collection must be structured to allow gradual refinement through selection of more specific profiles as information about the user becomes available. Once created these user profiles need to be hard-coded and stored into the implementation. Additionally, such a volume of complex data structures has the potential to place a significant computational load on the system, requiring it to store, sort, organise and match in real-time after each interaction with the user.

Once the profiles have been designed, created, written and implemented they are difficult to change or update. Should changes be made to the general structure of the profile, for example by adding a new user characteristic, all profiles need to be adjusted to reflect that change. Maintaining an understanding of the structure of the profile collection could also become difficult if the system increases in scope. For a system applied to a small area, for example visitors to a museum, the number of user profiles is bounded by the known types of visitors but if the system expands to new audiences then new profiles need to be written and fitted into the system. At present the collection of profiles and their relation to each other needs to be crafted by a human designer, entailing a significant effort and increasing the difficulties involved in expanding or updating the system.

Another side effect of using a collection of unchanging user profiles is the difficulty in incorporating known information about a user. While the model's approach is to combine in some way the user information, contained in the Visitor Profile with the chosen user profile, it is unclear as to how this can be achieved. By combining the two profiles in this way a new profile is created. No information about the design impact of this profile would be available since the rules associated with a stored user profile would not apply in this instance. Further work needs to be done to ensure that the presentations generated are truly personalised rather than merely applying a matched stereotype regardless of how effective the matching process may be.

A possible alternative to using complete user profiles with design impacts would be to use individual characteristics. This would have the advantage of allowing more flexibility in updating and expanding the way in which a user is described and in incorporating specific user information into the profile. In this way profiles could be created from lists of characteristics to find the design decisions for the user. However, this has the disadvantage of ignoring the effect of combinations of characteristics and reduces the effectiveness of the profile solution for addressing the lack of information problem. Separating the characteristics means separating their impacts which will result in loss of potential information and inferences possible from combined knowledge unless that combined effect is written in as well. User profiles allow the system to deduce information to fill gaps in that available from the user which using only individual characteristics does not do. The best solution may lie somewhere between these two possibilities, combining a collection of user profiles with information on individual effects.

The requirement for a complete (or near complete) collection of user profiles while contributing to, at least, a partial solution for the problem of lack of information about a user, is a distinct disadvantage of Loeber's approach since it requires a lot of human effort to create and implement the profiles and is difficult to expand and inflexible. However, for real systems based upon known target audiences and having a restricted scope, this has the potential to provide a workable solution.

\subsection{Progressive Adaptation based on User Navi- gation}

The companion tool to address the problem of lack of information is to utilise the user's navigation path to allow the profile to be refined. When applied to a large, structured and pre-designed web site this approach has great potential. However, automatically generated hypermedia presentations may pose some serious problems. The structure and interactivity of the presentation is unknown at design time. In fact the structure is influenced by the user model. In order to refine the user profile applied to the visitor, their path through the presentation is used but if the possible paths are unknown then predictions of the user's purpose when making particular choices cannot be made. Additionally in the case of some multimedia presentations the level of interaction may be such that the visitor becomes more of an audience than an interactive participant — their user profile may even encourage this. Once this interaction is reduced then the opportunities for the system to make inferences about the user from their behaviour is lost and with it the adaptability of the user profiles.

While in theory the adaptation of an interface based on the user's navigation appears to provide a mechanism to deal with limited available user data, evaluation of its impact upon the user is required. Consistency is a byword for interface designers and HCI researchers alike and progres- 
sive adaptation, resulting in changing parameters for the user, creates inconsistency in the interaction and possibly reduces the support for the user. This could well negate any benefits gained from the adaptive approach.

Applying real-time, progressive adaptation to multimedia also raises other questions. As has already been discussed navigation and interaction through a web site is potentially very different to navigation through a multimedia presentation. The adaptation depends on the concept of a page which can be created or modified and served to the user. In multimedia there is no agreed upon equivalent definition of this concept. One suggestion has been to classify the segment of information returned from the server as a multimedia 'page'. A page could therefore be defined by the user's clicks. While this seems to be a sensible and workable definition, it results in a widely varying pace of adaptation, increasing the potential for user confusion. The transient nature of the relationship with the user that may occur at a web site whose sole purpose is to generate onetime-only presentations is also likely to reduce the effectiveness of the progressive adaptation.

Overall, it is unclear how effective adaptation and refinement of the user profile based on navigation choices can be. On one hand, it utilises an excellent source of available information about the user to provide support for the choice of user profile while on the other its effectiveness for the field of multimedia and its impact on the overall consistency of the interaction is yet to be determined. The solution seems to be to alter the focus of Loeber's model. Placing the presentation generated within the scope of a complete and interactive web site allows some of the advantages of analysing the user's path through the site and applying it to refinement of the profile to be realised. The approach can then be applied to the system as a whole rather than solely to the multimedia generation aspect.

\subsection{Design Decisions and MAO}

In the making of any design decision, whether it be by a human or a computer program, compromise is a term often used. When requirements from different participants are conflicting, choices need to be made to decide on the best solution. For example, a user likes a particular colour, orange, while the author of the presentation wants to use corporate colours, red and white, to present the company identity to the user. Depending on the particular style chosen for the presentation, this is likely to cause a clear conflict since both requirements cannot be satisfied at the same time. Another example could be if a design rule was implemented that aimed to provide a background colour which complemented the image being displayed. Not only could this be in conflict with the corporate colours desired by the author but knowledge about the make up of the content will be required before the rules can be applied and the decision made. These examples illustrate the decisions that must be made by an automatic generation system that require input from the user modelling section.

To overcome these difficulties, Loeber's solution is to apply the MAO concept. This gives a mechanism for comparison and ranking and therefore the ability to make a decision. The difficulty with using the impact of user's levels of motivation, ability and opportunity is that it is very much a subjective, human measure. Converting this into a concrete, numerical record capable of analysis and comparison by a computer program is difficult. In doing so much of the effectiveness is likely to be reduced. Yet this is a vital and necessary step before the optimisation layer of Loeber's model can be implemented and such conflicts and contradictory requirements be judged on their likely user impact. Once a method for recording and measuring MAO impact is found, rules describing the impact of implementing choices must be written, again by a human designer, for each of the user profiles.

The inter-dependencies between content, style and structure in multimedia is a perennial problem in automatic generation of presentations and creates extra complications when applying user preferences. Since user preferences impact all of these areas, in the choice of media items (content), the discourse used to present the items (structure) and the colour, font, speed etc. that the items are displayed by (style), the effect of user requirements is far reaching and complex. Simple, direct, cause-and-effect rules describing MAO impact may not be enough to cope with this level of complexity. The ramifications of any decision are more far reaching than to impact upon only one aspect of the content, style or structure of a presentation. Any decision made should also be analysed to determine its impact on all areas of the presentation. Loeber's approach relies on the adequate creation of rules associated with the profiles in the user model expert and the decision making abilities of the optimisation layer. The scalability of this approach, in coping with increasingly more complex implications of design decisions, is unclear.

This section has examined some of the problems inherent in applying user modelling to multimedia presentation generation in general and some potential issues with Loeber's approach in particular. These are the questions that needed to be pondered when integrating a prototype for user preferencing into the Cuypers system.

\section{Future and Ongoing Work}

The goal of this project was to lay the groundwork for the inclusion of user preferences in the Cuypers prototype. Section 2 describes the implementation of the framework necessary to achieve this. In order to complete the addition 
of user modelling to the Cuypers architecture some future work remains.

- Many of the 'fact' portions of the system require some further research and implementation. Facts including user profiles, design rules and abstract representations for style choices (eg. red is [HSL value]) need to be created and written.

- The composition of user profiles and the creation of a suitable collection must be done involving investigation into user models and how to retrieve information from a visitor.

- Design rules for each profile need to be created. This involves deciding the MAO impact for stylistic choices.

- Colour is the only currently implemented aspect. The mechanism for selecting suitable foreground and background colours is currently under development and as it progresses further it may result in alterations to other parts of the system

- Selection and matching techniques for other aspects (text, structure, speed etc) need to be written or incorporated into the existing selection method. By the nature of the structure implemented currently this should not present a problem but should slot into the existing architecture.

Some areas which may be worthy of further investigation with respect to matching the visitor with a suitable user profile are neural networks and work in fuzzy logic techniques [7]. The current matching system is sufficient but this may be due to the smaller scope of this project. Interesting applications of neural networking techniques to structure and match user profiles could be useful in this area but significant further work is required. Existing work on applying fuzzy logic techniques to classify an unknown user into an existing user class is also promising.

Finally, evaluation of the success of Loeber's model is required. This is ongoing and involves a significant amount of work but is very necessary to determine how successful the approach is. In particular the success in the matching user profiles algorithm and the decision making MAO expert should be determined. More generally the impact of progressive adaptation on the user, both for multimedia and the web in general, should be evaluated and the overall result of utilising MAO in user modelling.

\section{Conclusion}

One of the goals of this project was to investigate the practical application of the user model developed by Susanne Loeber. As a result of this, some conclusions and recommendations for the further development of the model have been reached.

Firstly, the issues with user profiles, as discussed in section 3.1, need to be addressed. Most importantly the question of incorporating specific, individual information about the visitor with existing stored user profiles. If this is possible then it will also, effectively, reduce the number of stored profiles required and simplify their structure and organisation.

Secondly, evaluation is required on the effect of progressive adaptation on the user and how it can be achieved in multimedia presentations. Section 3.2 discusses some of the issues with this approach. Questions include finding the optimal rate of change and clearly defining what interaction means when applied to multimedia presentations.

Overall there is a need to clearly define the focus and scope of the model. If, as indicated by its combination with the SRM IMMPS, it is applied to multimedia presentation generation then the problems raised in sections 3.2 and 3.3 need to be addressed. Specifically, the use of the navigation path, which is itself generated by the profile chosen, to select an appropriate user profile.

In conclusion, this project sought to investigate the application of Loeber's model to a real system and to incorporate user influence into Cuypers. This has been achieved by implementing the framework of Loeber's approach, the Design Expert, User Model Expert and Optimisation Layer (as MAO Expert), and using the sample of colour choices. The architecture now in place has the potential to be extended and applied to other areas such as text size and style, speed and structure. This has increased the capacity for generating personalised multimedia presentations and has demonstrated some of the benefits and potential issues with Loeber's approach to user modelling.

\section{Acknowledgements}

Thanks to Lynda Hardman (CWI, Amsterdam supervisor) and Jane Hunter (DSTC, Brisbane supervisor) for arranging my visit. To Susanne Loeber for her insights and help with understanding user modelling. My colleagues at CWI, for their comments and assistance, especially to Amit Manniesing and Katharina Schwarz for their helpful discussions on user modelling and design rules. This work was conducted under the NWO NASH project and travel to Amsterdam was funded by DSTC.

\section{References}

[1] M. Bordegoni, G. Faconti, M. Maybury, T. Rist, S. Ruggieri, P. Trahanias, and M. Wilson. A Standard Reference Model for Intelligent Multimedia Presentation Systems. Computer Standards \& Interfaces, 18(6-7):477-496, December 1997. 
[2] P. Brusilovsky and D. W. Cooper. Domain, Task and User Models for an Adaptive Hypermedia Performance Support System. In Intelligent User Interfaces, IUI'02, January 2002.

[3] M. B. Coney and M. Steehouder. Role playing on the web: guidelines for designing and evaluation personas online. Technical Communications, 3rd quarter:327-340, 2000.

[4] A. Csinger, K. S. Booth, and D. Poole. AI Meets Authoring: User Models for Intelligent Multimedia. In Artificial Intelligence Review, 1994.

[5] J. Fink, A. Kobsa, and A. Nill. Towards a user-adapted information environment on the Web. In Multimedia and Standardization 98, Paris, France, 1998.

[6] W. D. Hoyer and D. McInnes. Consumer Behaviour. Houghton Mofflin Company, 2nd edition edition, 2001.

[7] J.M.Rossiter, T.H.Cao, T.P.Martin, and J.F.Baldwin. User recognition in uncertain object oriented user modelling. In Proceedings of the 10th IEEE International Conference of Fuzzy Systems, FUZZ-IEEE 2001, 2001.

[8] A. Kobsa, J. Koenemann, and W. Pohl. Personalized Hypermedia Presentation Techniques for Improving Online Customer Relationships. In The Knowledge Engineering Review, volume 16, pages 111-155, 2001.

[9] A. Kobsa, D. Muller, and A. Nill. KN-AHS: An Adaptive Hypertext Client of the User Modeling System BGP-MS. In Proceedings of the Forth International Conference on User Modeling, pages 99-105, 1994.

[10] A. Kobsa and W. Pohl. The User Modeling Shell System BGP-MS. User Modeling and User=Adapted Interaction, 4(2):59-106, 1995.

[11] A. Kobsa and W. Wahlster, editors. User Models in Dialog Systems. Springer-Verlag, 1989.

[12] S. Loeber. Modelling the audience of a web-based presentation: context-driven, rhetorical role-playing approach. In ecce11, unknown 2002.

[13] S. Loeber and A. Cristea. A WWW Information-Seeking Process Model. In The 8th International Conference of the International Society for the Study of European Ideas, July 2002.

[14] S. G. Loeber, L. M. Aroyo, and L. Hardman. An explicit model for tailor-made ecommerce web presentations. In unknown, unknown 2002.

[15] Microsoft Office Homepage. web page. http://www.microsoft.com/office/.

[16] E. Rich. Users are Individuals: individualizing user models. Int. J. Human-Computer Studies, 51:323-338, 1999.

[17] L. A. Suchman. The problem of Human-Machine Communication. Cambridge University Press, 1987.

[18] J. van Ossenbruggen, J. Geurts, F. Cornelissen, L. Rutledge, and L. Hardman. Towards Second and Third Generation Web-Based Multimedia. pages 479-488, 2001.

[19] J. van Ossenbruggen and L. Hardman. Multimedia on the Semantic Web. In H. van Oostendorp, A. Dillon, and L. Breure, editors, Creation, Use and Deployment of Digital Information. Erlbaum, Publication planned late 2002.

[20] J. van Ossenbruggen, L. Hardman, and L. Rutledge. Hypermedia and the Semantic web: A research agenda. Technical Report INS-R0105, CWI, 2001. 